\title{
Blefarospasmo essencial e espasmo hemifacial: características dos pacientes, tratamento com toxina botulínica A e revisão da literatura
}

\author{
Essential blepharospasm and hemifacial spasm:characteristic of the patient, \\ botulinum toxin A treatment and literature review
}

\author{
Silvana Artioli Schellini ${ }^{1}$ \\ Olívia Matai ${ }^{2}$ \\ Thais Zamudio Igami $^{3}$ \\ Carlos Roberto Padovani ${ }^{4}$ \\ Carlos Pereira Padovani ${ }^{5}$
}

Trabalho realizado na Faculdade de Medicina de Botucatu - Universidade Estadual Paulista "Julio Mesquita Filho" - UNESP - Botucatu (SP) - Brasil.

${ }^{1}$ Professora e Livre Docente do Departamento de Oftalmologia, Otorrinolaringologia e Cabeça e Pescoço da Faculdade de Medicina de Botucatu - Universidade Estadual Paulista "Julio Mesquita Filho" - UNESP Botucatu (SP) - Brasil.

Residente de Oftalmologia do Departamento de Oftalmologia, Otorrinolaringologia e Cabeça e Pescoço da Faculdade de Medicina de Botucatu - Universidade Estadual Paulista "Julio Mesquista Filho" - UNESP Botucatu (SP) - Brasil.

${ }^{3}$ Graduanda do Departamento de Oftalmologia, Otorrinolaringologia e Cabeça e Pescoço da Faculdade de Medicina de Botucatu - Universidade Estadual Paulista "Julio Mesquita Filho" - UNESP - Botucatu (SP) - Brasil.

${ }^{4}$ Professor Titular do Instituto de Biociências da Faculdade de Medicina de Botucatu - Universidade Estadual Paulista "Julio Mesquita Filho" - UNESP - Botucatu (SP) - Brasil.

${ }^{5}$ Mestrando pela Faculdade de Ciências Agronômicas da Universidade Estadual Paulista "Julio Mesquita Filho" - UNESP - Botucatu (SP) - Brasil.

Endereço para correspondência: Silvana Artioli Schellini. Dep. de Oftalmologia, Otorrinolaringologia e Cabeça e Pescoço - Faculdade de Medicina de Botucatu Universidade Estadual Paulista "Julio Mesquita Filho" (UNESP). Botucatu (SP) CEP 18618-970

E-mail: sartioli@fmb.unesp.br

Recebido para publicação em 30.03.2005

Versão revisada recebida em 22.08.2005

Aprovação em 27.10.2005

\section{RESUMO}

Objetivo: Conhecer as características dos portadores de blefarospasmo essencial e espasmo hemifacial, assim como a resposta ao tratamento utilizando toxina botulínica A. Métodos: Trinta e quatro portadores de blefarospasmo essencial ou espasmo hemifacial foram avaliados quanto a idade, sexo, queixas oculares, tempo de existência da doença, tipo de comprometimento, complicações e resultado do tratamento com toxina botulínica A. Resultados: A mediana da idade dos pacientes foi de 63 anos e a média, de 61 anos, sem diferença quanto ao sexo; $66,66 \%$ possuíam espasmo hemifacial e 33,33\%, blefarospasmo essencial. Vários pacientes apresentavam também olho seco. A melhora com a utilização da toxina botulínica A ocorreu em 91,30\% dos pacientes tratados. As complicações com o tratamento foram ptose palpebral $(8,33 \%)$ e desvio da rima bucal $(8,33 \%)$. Conclusão: O blefarospasmo essencial e o espasmo hemifacial geralmente acometem idosos, de ambos os sexos. O tratamento com a toxina botulínica A é eficiente, com índice muito baixo de complicações.

Descritores: Blefaroespasmo/quimioterapia; Espasmo hemifacial/quimioterapia; Toxina botulínica tipo A/uso terapêutico; Potenciais de ação; Músculos oculomotores

\section{INTRODUÇÃO}

O mecanismo fisiológico do blefarospasmo (espasmo do orbicular) e da síndrome de Meige (afeta também a face inferior e pescoço) ainda não é conhecido $^{(1)}$. O blefarospasmo essencial e os espasmos faciais foram muitas vezes confundidos com doenças oculares ou distúrbios psíquicos ${ }^{(2)}$. Apesar do blefarospasmo não implicar em alterações oculares, algumas doenças dos olhos podem desencadeá-lo ${ }^{(3)}$ e lesões oculares podem preceder o quadro, o que ocorre em cerca de $12,1 \%$ dos $\operatorname{casos}^{(4)}$.

As alterações psíquicas, como comportamento obsessivo compulsivo ${ }^{(5)}$ e esquizofrenia ${ }^{(6)}$ podem estar presentes em portadores de blefarospasmo essencial.

Apesar do blefarospasmo essencial estar presente em indivíduos sem qualquer alteração neurológica, disfunção de gânglios da base ${ }^{(5)}$ ou lesão em núcleos da base, observadas por ressonância nuclear magnética ${ }^{(2)}$, já foram relatadas.

O blefarospasmo pode ser desencadeado por medicamentos, como os neurolépticos ${ }^{(7)}$ e pode haver predisposição genética em 9,5\% ${ }^{(4)}$. É caracterizado por movimentos involuntários das pálpebras, por vezes com dificuldade de abertura palpebral (apraxia). 
O espasmo hemifacial é caracterizado por contrações tônicas e clônicas dos músculos inervados pelo facial ipsilateral. É importante distinguir o espasmo hemifacial do espasmo psicogênico, tics, mioquimia, blefarospasmo e discinesia tardia. Ressonância magnética pode mostrar compressão vascular da raiz do $7^{\circ}$ nervo ${ }^{(8)}$.

O tratamento do blefarospasmo essencial e do espasmo hemifacial passou por várias fases. Entretanto, desde o ano de 1984, quando se usou a toxina botulínica A em alguns pacientes já submetidos a cirurgia com boa resposta e duração média do efeito de 10 semanas, com diminuição da força muscular em cerca de $10 \%^{(9)}$ e também em pacientes resistentes a outros tratamentos, com bons resultados e com efeitos colaterais locais, pequenos e transitórios ${ }^{(10)}$, este é o tratamento mais empregado para este fim; atualmente a toxina botulínica é considerada o melhor tratamento para o blefarospasmo essencial e síndrome de Meige ${ }^{(11)}$, a ponto de se dizer: "Botulinum isn't just a poison anymore"(12).

A toxina botulínica age por bloqueio seletivo e reversível da junção neuro-muscular, causando relaxamento da musculatura envolvida ${ }^{(13)}$. A toxina inibe a liberação de acetilcolina das vesículas pré-sinápticas da placa neural, provocando uma neurectomia transitória ${ }^{(14)}$.

O presente estudo foi desenvolvido com o objetivo de conhecer as características dos portadores de blefarospasmo essencial e espasmo hemifacial, assim como a resposta ao tratamento com toxina botulínica A, em nosso meio.

\section{MÉTODOS}

Foram estudados 34 portadores de blefarospasmo essencial ou espasmo hemifacial, atendidos no Serviço de Plástica Ocular da Faculdade de Medicina de Botucatu - UNESP, de 1999 até 2003, avaliando-se: idade, sexo, queixas oculares, tempo de existência da doença, tipo de comprometimento, tratamento efetuado, complicações e resultado do tratamento.

O tratamento empregado foi a toxina botulínica tipo A, tendo se utilizado o Botox $^{\circledR}$ (100U) (diluição de 1 ampola em $4 \mathrm{ml}$ de soro fisiológico, com injeção de $0,1 \mathrm{ml}$ por sítio) ou o Dysport $^{\circledR}$ (500 U) (diluição de 1 ampola em 2,5 ml de soro fisiológico, com injeção de $0,1 \mathrm{ml}$ por sítio). As injeções foram aplicadas na musculatura orbicular, na região imediatamente superior ao supercílio e na pálpebra inferior. No caso do espasmo hemifacial, também outros sítios receberam aplicação, conforme a necessidade de cada paciente.

Os dados obtidos foram submetidos a análise estatística descritiva, teste do Qui-quadrado para uma amostra e teste de Fisher.

\section{RESULTADOS}

A mediana de idade dos 34 pacientes foi de 63 anos e a média, de 61 anos (Tabela 1). Quanto ao sexo, os pacientes não diferiram $\left(X^{2}=0,12(p>0,05)\right)$. Dos portadores, $66,66 \%$ possuíam espasmo hemifacial e 33,33\%, blefarospasmo essencial $\left(X^{2}=3,00(p>0,05)\right)$.

Os pacientes, além dos movimentos involuntários nas pálpebras, apresentavam queixas compatíveis com olho seco $\left(\mathrm{X}^{2}=34,25(\mathrm{p}<0,0001)\right)$.

O problema estava presente há menos de 1 ano $(22,58 \%)$, de 1 a 5 anos $(32,26 \%)$ ou mais do que 5 anos $(16,13 \%)$, sem diferença estatística $\left(X^{2}=1,90(p>0,05)\right)$.

A melhora com a utilização da toxina botulínica A ocorreu em $91,30 \%$ dos pacientes tratados $\left(X^{2}=15,70(p<0,0001)\right)$.

Com este tipo de utilização, observaram-se complicações como: ptose $(8,33 \%)$ e desvio da rima $(8,33 \%)\left(\mathrm{X}^{2}=27,00\right.$ $(\mathrm{p}<0,0001))$. A maioria dos pacientes não apresentou reações adversas.

\section{DISCUSSÃO}

O uso de toxina botulínica está se ampliando nos últimos anos, sendo utilizada para tratamento de blefarospasmo, espasmo hemifacial, estrabismo, distonias, espasticidade muscular, tremores, aplicações cosméticas, "migraine", cefaléia tensional, distonia oromandibular e cervical, proctalgia, disfagia, gastroparesia, espasticidade cerebral, nistagmo, entrópio espástico, distonias $^{(14-16)}$, hiperidrose palmar, axilar ou plantar ${ }^{(13)}$. O tratamento de linhas hiperfuncionais da face está sendo feito desde $1992^{(17)}$, aumentando exponencialmente ${ }^{(18)}$.

Este estudo se refere à utilização desta medicação em portadores de blefarospasmo essencial e espasmo hemifacial. A média de idade dos pacientes foi de 61 anos, semelhante ao observado por outros ${ }^{(4)}$, cujos pacientes apresentavam média de 55,8 anos.

Homens e mulheres tiveram a mesma chance de acometimento, ao contrário do que foi observado por outros que tiveram maior acometimento em mulheres $(1,8 / 1,0)^{(4)}$.

A queixa de olho seco foi bastante freqüente dentre os pacientes aqui estudados, tendo sido esta associação já relatada por outros, que referiram que o blefarospasmo melhora após a administração da toxina, mas que o olho seco pode até piorar $^{(19)}$. Parece que o olho seco, lacrimejamento e ptose transitória ocorrem mais em indivíduos que já tiveram cirurgia prévia de pálpebra ${ }^{(20)}$.

É comum que o indivíduo com blefarospasmo apresente também fotofobia, o que se explica por estimulação simpática ${ }^{(21)}$.

Tabela 1. Distribuição dos portadores de blefarospasmo e espasmo hemifacial conforme faixa etária

\begin{tabular}{lccccc} 
Faixa etária & $31-\mathbf{4 5}$ & $46-60$ & $\mathbf{6 1}-\mathbf{7 5}$ & $>75$ & Total \\
Número de pacientes & $4(13,3 \%)$ & $7(23,3 \%)$ & $13(43,4 \%)$ & $5(16,7 \%)$ \\
*Idade de quatro pacientes não informada & & & $30 *(100 \%)$ \\
\hline
\end{tabular}


O problema pode estar presente há vários anos, como foi observado na amostra estudada. Também se observou o espasmo hemifacial como mais freqüente que o blefarospasmo essencial.

O tratamento empregado foi a toxina botulínica $\mathrm{A}$, tendo sido utilizado o Botox ${ }^{\circledR}$ em alguns pacientes e, em outros, o Dysport $^{\circledR}$, sem que houvesse uma seleção prévia da medicação a ser empregada.

Existem diferenças entre a toxina de origem inglesa (Dysport $^{\circledR}$ ) e americana (Botox ${ }^{\circledR}$ ). A concentração das medicações é diferente, o que leva a necessidade de diluição de forma diversa. Utiliza-se no Serviço a diluição de 4:1 para o Botox e de 2,5:1 do Dysport. Desta forma, aplicou-se em média o total de $1 \mathrm{ml}$ de substância na região periocular dos pacientes que apresentavam o problema bilateralmente.

Um estudo comparou ambas as drogas em diluição de 4 $\left(\right.$ Botox $\left.^{\circledR}\right): 1$ (Dysport ${ }^{\circledR}$ ) e foi visto que o Dysport tem efeito médio por 13 semanas e o Botox, por 11. Os efeitos advsersos foram notados em $47 \%$ com Botox e 50\% com Dysport ${ }^{(22)}$. Portanto, ambas as drogas possuem efeito semelhante, da mesma forma que nos pareceu quando utilizadas nos nossos pacientes.

A dose a ser empregada difere entre os autores. Bons resultados foram relatados usando-se 12,5 unidades de Dysport $^{\circledR}$ por aplicação, a cada lado ${ }^{(23)}$.

As drogas foram utilizadas na região superior do supercílio. Porém, também foi sugerida a injeção pré-tarsal (no músculo de Riolan, na extremidade medial e lateral da pálpebra superior) e pré-septal, reportando-se como vantagens a necessidade de quantidade menor da droga ${ }^{(24)}$, com poucos efeitos colaterais $^{(25-27)}$, tais como a ptose palpebral.

Apesar dos relatos sobre a necessidade de se aumentar em cerca de $50 \%$ a quantidade da droga a ser administrada com o passar do tempo ${ }^{(28)}$, os pacientes deste estudo continuam respondendo a mesma quantidade de medicamento usada no início do tratamento.

A eficácia do tratamento é alta. Os resultados aqui obtidos mostraram que a toxina botulínica foi efetiva para $91,3 \%$ dos pacientes. Para outros, a eficácia esteve por volta de $70 \%{ }^{(29)}$, $85 \%{ }^{(30-31)}$ e até $97 \%$ dos pacientes ${ }^{(32)}$. A chance de melhora com o tratamento está também relacionada com o tipo de problema que o paciente apresenta, sendo pior nas distonias ${ }^{(29)}$.

Não foi aqui avaliado o intervalo de duração do efeito. A nossa conduta tem sido reaplicação da medicação a cada 90 dias (em média), não se tendo como rotina a avaliação do efeito neste momento. Porém, sabe-se que a droga pode ter duração do efeito de 2,6 até 4,3 meses $^{(23)}$, ou até 170 dias $^{(20)}$. A duração difere com relação ao problema apresentado, de forma que para o blefarospasmo a duração pode ser de até 24 semanas e para o espasmo hemifacial, de $32^{(30)}$. Também a severidade do quadro influencia a duração do tratamento ${ }^{(33)}$.

A resposta ao tratamento pode ter intensidades diferentes, podendo-se utilizar a escala de Jankovic para quantificar a resposta ao tratamento com a toxina ${ }^{(34)}$.

As complicações mais freqüentes nos nossos pacientes foram a ptose palpebral e o desvio da rima bucal. A complicação mais freqüentemente relatada é mesmo a ptose $(3,8 \%)^{(31)}$, podendo-se também ter diplopia, fraqueza das pálpebras, disfagia e pneumonia aspirativa (na síndrome de Meige) ${ }^{(29)}$, fraqueza na musculatura da face, vômito, edema e/ou equimose ${ }^{(31)}$.

Apesar de não se ter observado resistência ao tratamento, este fato existe em cerca de $10 \%$ dos pacientes e outras toxinas que não a toxina A tem sido empregadas, como a toxina botulínica sorotipo $\mathrm{F}^{(33)}$ e a toxina $\mathrm{B}^{(35)}$.

Levantamento feito por meio de questionários, aplicado à 1.653 portadores de blefarospasmo, mostrou que a toxina, assim como o tratamento cirúrgico, dão bons resultados $87 \%$ de melhora com miectomia total e $96 \%$ com toxina; o uso combinado de miectomias parciais, associadas com a administração da toxina, fornece excelentes resultados ${ }^{(32)}$.

Apesar da toxina botulínica ser bastante efetiva e de melhorar a qualidade de vida dos portadores de blefarospasmo e espasmo hemifacial ${ }^{(36)}$, o custo é ainda considerado alto ${ }^{(36-37)}$. O Dysport ${ }^{\circledR}$ possui concentração recomendada pelo fabricante de $50 \mathrm{U} / \mathrm{ml}$. Porém, acrescentando-se albumina abaixa-se sua concentração em cerca de $30 \%$, com o mesmo efeito e com redução do custo e menor chance de desenvolver anticorpos ${ }^{(38)}$.

A avaliação da qualidade de vida por questionário com o uso da toxina botulínica revelou melhora, mas acompanhada do medo de que a medicação perca efeito e depressão ${ }^{(39)}$.

Apesar da remissão da doença ser possível, não foi por nós observada ${ }^{(40)}$.

Tendo em vista a alta eficácia, os poucos e transitórios efeitos colaterais e a segurança de aplicação( ${ }^{(41)}$, mesmo a longo prazo $^{(42)}$, acredita-se ser esta droga ainda de larga utilização no arsenal de tratamento do blefarospasmo e do espasmo hemifacial.

\section{CONCLUSÃO}

O blefarospasmo e o espasmo hemifacial geralmente acometem idosos, de ambos os sexos. O tratamento com a toxina botulínica é eficiente, com índice muito baixo de complicações.

\section{ABSTRACT}

Purpose: To evaluate the characteristics of the essential blepharospasm and hemifacial spasm patients and the feasible treatment with botulinum toxin A. Methods: Thirty-four essential blepharospasm or hemifacial spasm patients were evaluated according to gender, ocular complaint, time of disease, treatment outcome and complications. Results: Age median was 63 years and the mean was 61 years, with no difference regarding sex; $66.66 \%$ of the patients had hemifacial spasm and $33.33 \%$, essential blepharospasm. Many patients complained of dry eye associated with involuntary spasm. Botulinum toxin A showed a positive outcome in $91.30 \%$ of the treated patients and complications observed after treatment were eyelid ptosis $(8.33 \%)$ and buccal angle deviation ( $8.33 \%)$. Conclusion: Essential blepha- 
rospasm and hemifacial spasm occurred in the elderly, of both sexes. Treatment with botulinum toxin A was useful, with very low complication rates.

Keywords: Blepharospasm/drug therapy; Hemifacial spasm/ drug therapy; Botulinum toxin type A/therapeutic use; Action potentials; Oculomotor muscles

\section{REFERÊNCIAS}

1. Faulstich ME, Carnrike CL Jr, Williamson DA. Blepharospasm and Meige syndrome: a review of diagnostic, aetiological and treatment approaches. J Psychosomat Res. 1985;29(1):89-94.

2. Shapiro RL, Hatheway C, Swerdlow DL. Botulism in the United States: a clinical and epidemiologic review. Ann Intern Med. 1998;129(3):221-8.

3. Elston JS, Marsden CD, Grandas F, Quinn NP. The significance of ophthalmological symptoms in idiopathic blepharospasm. Eye. 1998;2(Pt 4):435-9.

4. Grandas F, Elston J, Quinn NP, Marsden CD. Blepharospasm: a review of 264 patients. J Neurol Neurosurg Psychol. 1998;51(6):767-72.

5. Broocks A, Thiel A, Angenstein D, Dressler D. Higher prevalence of obsessivecompulsive symptoms in patients with blepharospasm than in patients with hemifacial spasm. Am J Psychiatry. 1998;155(4):555-7.

6. Sachdev P. Tardive blepharospasm. Mov Disord. 1998;13(6):947-51.

7. Levin H, Reddy R. Clozapine in the treatment of neuroleptic-induced blepharospasm: a report of 4 cases. J Clin Psychol. 2000;61(2):140-3.

8. Tan NC, Chan LL, Tan EK. Hemifacial spasm and involuntary facial movements. QJM. 2002;95(8):493-500.

9. Frueh BR, Felt DP, Wojno TH, Musch DC. Treatment of blepharospasm with botulinum toxin. A preliminary report. Arch Ophthalmol. 1984;102(10):1464-8.

10. Tsoy EA, Buckley EG, Dutton JJ. Treatment of blepharospasm with botulinum toxin. Am J Ophthalmol. 1985;99(2):176-9.

11. Mauriello JA Jr, Dhillon S, Leone T, Pakeman B, Mostafavi R, Yepes MC Treatment selections of 239 patients with blepharospasm and Meige syndrome over 11 years. Br J Ophthalmol. 1996;80(12):1073-6.

12. Cibis GW, Waeltermann J. Botulinum isn't just a poison anymore. Botulinum toxin in treatment of strabismus and essential blepharospasm. Mo Med. 1985; 82(3):145-7.

13. Kohler A, Ochsner F. Current indications for the treatment with botulinum toxin. Rev Med Suisse Romande. 2001;121(6):471-4.

14. Zajacz M. [Applications of the botulinum A toxin]. Orvosi Hetilap. 2003; 144(18):837-42. Hungarian.

15. Johnson EA. Clostridial toxins as therapeutic agents: benefits of nature's most toxic proteins. Annu Rev Microbiol. 1999;53:551-75.

16. Singer C. [Indications and management of botulinum toxin]. Rev Neurol. 1999; 29(2):157-62. Spanish.

17. Sarrabayrouse MA. Indications and limitations for the use of botulinum toxin for the treatment of facial wrinkles. Aesthetic Plast Surg. 2002;26(4):233-8.

18. Binder WJ, Blitzer A, Brin MF. Treatment of hyperfunctional lines of the face with botulinum toxin A. Dermatol Surg. 1998;24(11):1198-205.

19. Horwath-Winter J, Bergloeff J, Floegel I, Haller-Schober EM, Schmut O. Botulinum toxin A treatment in patients suffering from blepharospasm and dry eye. Br J Ophthalmol. 2003;87(1):54-6.

20. Scott AB, Kennedy RA, Stubbs HA. Botulinum A toxin injection as a treatment for blepharospasm. Arch Ophthalmol. 1985;103(3):347-50.
21. McCann JD, Gauthier M, Morschbacher R, Goldberg RA, Anderson RL, Fine PG, et al. A novel mechanism for benign essential blepharospasm. Ophthal Plast Reconstr Surg. 1999;15(6):384-9.

22. Sampaio C, Ferreira JJ, Simões F, Rosas MJ, Magalhães M, Correia AP, et al. Dysbot: a single blind, randomized parallel study to determine whether any differences can be detected in the efficacy and tolerability of two formulations of botulinum toxin type A - Dysport and Botox - assuming a ratio of 4:1. Mov Disord. 1997;12(6):1013-8.

23. Shorr N, Seiff SR, Kopelman J. The use of botulinum toxin in blepharospasm. Am J Ophthalmol. 1985;99(5):542-6.

24. Mackie IA. Riolan's muscle: action and indications for botulinum toxin injection. Eye. 2000;14(Pt 3A):347-52.

25. Jankovic J. Pretarsal application of botulinum toxin for treatment of blepharospasm. J Neurol Neurosurg Psychol. 1995;59(3):309-11.

26. Jankovic J. Pretarsal application of botulinum toxin for blepharospasm and apraxia of eyelid opening. J Neurol Neurosurg Psychol. 1996;60(6):704-7.

27. Cakmur R, Ozturk V, Uzunel F, Donmez B, Idiman F. Comparison of preseptal and pretarsal injections of botulinum toxin in the treatment of blepharospam and hemifacial spasm. J Neurol. 2002;249(1):64-8.

28. Snir - Young JDH. Handbook of Botulinum Toxin Treatment. Br J Ophthalmol. 1996;80(9):854.

29. Andrade LA, Borges V, Ferraz HB, Azevedo-Silva SM. [Botulinum toxin A: experience in the treatment of 115 patients]. Arq Neuropsiquiatr. 1997;55(3B): 553-7. Portuguese.

30. Chang LB, Tsai CP, Liao KK, Kao KP, Yuan CL, Yen DJ, et al. Use of botulinum toxin $\mathrm{A}$ in the treatment of hemifacial spasm and blepharospasm. Zhonghua Yi Xue Za Zhi (Taipei). 1999;62(1):1-5.

31. Wang J, Lin S, Zhang X. [Blepharospasm and hemifacial spasm treated with botulinum A toxin injection]. Zhonghua Er Bi Yan Hou Ke Za Zhi. 1998;33(5): 291-3. Chinese

32. Anderson RL, Patel BC, Holds JB, Jordan DR. Blepharospasm: past, present and future. Ophthal Plast Reconstr Surg. 1998;14(5):305-17.

33. Chen RS, Lu CS, Tsai CH. Botulinum toxin A injection in the treatment of hemifacial spasms. Acta Neurol Scand. 1996;94(3):207-11.

34. Thussu A, Barman CR, Prabhakar S. Botulinum toxin treatment of hemifacial spasm and blepharospasm: objective response evaluation. Neurol India. 1999; 47(3):206-9.

35. Cardoso F. [Botulinum toxin type B in the management of dystonia nonresponsive to botulinum toxin type A]. Arq Neuropsiquiatr. 2003;61(3A): 607-10. Portuguese.

36. Osaki MH, Belfort Jr R. Qualidade e vida e custos diretos em pacientes com blefarospasmo essencial e espasmo hemifacial, tratados com toxina botulínica. Arq Bras Oftalmol. 67(1):43-9.

37. Schnider P, Birner P, Moraru E, Auff E. [Long-term treatment with botulinum toxin: dosage, treatment schedules and costs]. Wiener Klin Wochensch. 1999; 111(2):59-65. German.

38. Bigalke H, Wohlfarth K, Irmer A, Dengler R. Botulinum A toxin: Dysport improvement of biological availability. Exp Neurol. 2001;168(1):162-70.

39. Tucha O, Naumann M, Berg D, Alders GL, Lange KW. Quality of life in patients with blepharospasm. Acta Neurol Scand. 2001;103(1):49-52.

40. Bradley EA, Hodge DO, Bartley GB. Benign essential blepharospasm among residents of Olmsted County, Minnesota, 1976 to 1995: an epidemiologic study. Ophthal Plast Reconstr Surg. 2003;19(3):177-81.

41. Cunha MC, Aguirre OP, Souza-Dias CR. Tratamento do espasmo facial unilateral com toxina botulínica tipo A. Arq Bras Oftalmol. 1998;61(1):54-60.

42. Calace P, Cortese G, Piscopo R, Della Volpe G, Gagliardi V, Magli A, et al. Treatment of blepharospasm with botulinum neurotoxin type A: long-term results. Eur J Ophthalmol. 2003;13(4):331-6. 